\title{
Dissociative Identity Disorders in Korea: Two Recent Cases
}

\author{
Ilbin Kim, Daeho Kim ${ }^{凶}$, and Hyun-Jin Jung \\ Department of Psychiatry, Hanyang University College of Medicine, Seoul, Republic of Korea
}

\begin{abstract}
Although dissociative identity disorder (DID), the most severe of the dissociative disorders, has retained its own diagnostic entity since its introduction in the DSM-III, cases of DID are rarely seen in South and East Asia, likely due to the higher prevalence of possession disorder. We report two patients with DID who were recently admitted to our inpatient psychiatric unit and demonstrated distinct transitions to several identities. Their diagnoses were confirmed through a structured interview for dissociative disorders and possible differential diagnoses were ruled out by psychological, neuroimaging, and laboratory tests. The rapid transition to a Westernized, individualized society along with an increase in child abuse, might contribute to an increase in DID, previously under-diagnosed in this region.

Psychiatry Investig 2016;13(2):250-252
\end{abstract}

Key Words Dissociative identity disorder, Dissociative disorders, Culture, Asia, Childhood trauma.

\section{INTRODUCTION}

Dissociative identity disorder (DID) is rarely reported in Asia. It is so uncommon that some authors have speculated that DID is a culture-bound syndrome primarily found within Euro-American cultures. ${ }^{1}$ In fact, DID does appear in Asian countries, though its prevalence is much lower than that in Western countries. The prevalence of DID in the clinical population ranges 1 to $5 \%$ in North America, Europe, and Turkey, ${ }^{2}$ but only 0 to $0.5 \%$ in India, Bangladesh, and China. ${ }^{3-5}$ Only two case studies of DID have been reported in academic journals of South Korea. ${ }^{6,7}$ Here, we report two recent cases of Korean patients who experienced multiple childhood traumas and displayed several alternate identities.

\section{CASE}

\section{Case 1}

A 20-year-old Korean male was transferred from the military hospital and admitted to an intensive psychiatric ward due to complaints of episodic violent behaviors toward fellow

Received: April 6, 2015 Revised: June 17, 2015

Accepted: June 18, 2015 Available online: January 11, 2016

$\triangle$ Correspondence: Daeho Kim, MD, PhD

Department of Psychiatry, Hanyang University College of Medicine, 222 Wangsimni-ro, Seongdong-gu, Seoul 04763, Republic of Korea

Tel: +82-2-2290-8430, Fax: +82-2-2298-2055

E-mail: dkim9289@hanyang.ac.kr

(a) This is an Open Access article distributed under the terms of the Creative Commons Attribution Non-Commercial License (http://creativecommons.org/licenses/by$\mathrm{nc} / 3.0$ ) which permits unrestricted non-commercial use, distribution, and reproduction in any medium, provided the original work is properly cited. soldiers and changes in character and personality, which began at the onset of his military service two months earlier. Four months prior to admission, the patients returned home after six years of study abroad. His parents reported that the patient's behaviors differed from those of a visit one year prior, when he was shy and timid. After his return to Korea, the man appeared very confident and told his parents he would fare well in military service. In addition, he was very forgetful, often losing his belongings. The patient was on one occasion found by police in an alley far from his house, and he could not remember how he got there. Shortly after these incidents, the patient reported to the army for mandatory military service. At training camp, he seemed passionate and outgoing, quite different from his usual identity.

On several occasions, he only spoke English, which is not his native language. One incident involved a violent assault, in which the man injured another soldier. Alarmed by his emergent psychiatric problems, the military sent him to a psychiatric unit, where the staffs observed several different personalities. After one week, the military psychiatric staff decided to send him to a specialized civilian psychiatric hospital.

During the course of one month of hospitalization, seven alters were observed. The main host was very quiet and intimidated. John, an arrogant and uncooperative personality speaking only English, appeared on several occasions during the interviews. Another violent alter appeared twice when the patient thought of his childhood. During the transition to the violent alter, the patient broke a window with his fist and tried to hit his physician. Another alter named Cho appeared once. 
Cho introduced himself as the patient's story watcher and teller. Cho insisted he knew the host and other alters very well. He spoke both Korean and English, depicting the host as a "pity thing," the violent as a "thirsty killer," and the arrogant alter, John, as a "shit." While talking, Cho produced automatic writing with his left hand, of which he did not seem to be aware. We observed an additional three alters including a five-yearold boy, a mother-like personality, and a "metro-sexual swagger" alter. Cho was aware of the transition processes and he emphasized that there were more identities, although they were not observed during the hospitalization.

Laboratory tests including drug use screening and brain computerized tomography (CT) scan indicated no abnormalities of the host ego. Electrocardiogram (ECG) indicated "normal sinus rhythm." Interestingly, when retested as an alter, "Cho", the ECG indicated a "right bundle branch block." Cognitive impairment or malingering was ruled out through a full psychological battery, and no other psychiatric comorbidities were found. A structured clinical interview for DSM-IV dissociative disorder (SCID-D) confirmed the current diagnosis of DID.

In the SCID-D, the patient scored the severe category in amnesia, depersonalization, derealization, and identity confusion and identity alteration with additional fulfillment of mood change, age regression, and internal voice. He demonstrated the existence of alternate personalities with distinct names, ages, and character traits. Each alter took complete control of the patient's behavior, resulting in such occurrences as finding himself in a strange place, losing or discovering possessions in spots different from where he remembered leaving them, and speaking English. The patient also reported total amnesia during the domination of an alter.

The patient reported repeated childhood physical and emotional abuse and neglect by his parents. Each time he accessed these memories, he became agitated and transitioned to a violent alter. The treatment focused on stabilization, including affect regulation, grounding exercise and imagery techniques. Treatment made him more grounded and relaxed and decreased the frequency of alter emergence, particularly the violent alter. After one month of hospitalization, the patient was discharged.

\section{Case 2}

A 19-year-old female was admitted to our inpatient unit with episodic irritability and violence. These episodes first began one year prior to admission, after a severe conflict with her mother. The patient became agitated and panicked, with sweating and tremors; she then showed violent behaviors, including the destruction of household items and furniture. Thereafter, when she quarreled with others or her mother, the patient frequently experienced unrestrained irritability and violent behaviors. The patient did not recall these emotional episodes or behaviors.

During the course of hospitalization, four alters, including the host, were observed. Whenever the patient talked about childhood experiences, a 15-year-old girl named "Eunju" appeared. Before transitions, the patient experienced severe sweating and tremors. She did not recognize others, including her therapist and did not allow others near her. The patient was emotionally irritable and sobbed for nearly two hours in a typical sitting. She displayed a withdrawn posture before the transition back to her original identity, and had not recollection of her after. The patient also had a five-year-old-girl alter with childish speech. This alter began to appear relatively late during hospitalization, when the frequency of appearances of the violent alter gradually diminished. This young alter whines for her mother to come and comfort her at night. Another alter was a 30-year old woman with controlling nature. This alter was aware of the other identities, including the host ego, and expressed deep sympathy for their immaturity and suffering. Nevertheless, there were no signs of direct communication among the alters.

Laboratory tests including drug use screening and brain CT scan indicated no abnormalities of the host ego. Cognitive impairment or malingering was ruled out from a full psychological battery, and the patient met the criteria for diagnosis panic disorder with agoraphobia. The patient met diagnostic criteria of SCID-D, her symptoms falling into the severe category in amnesia, depersonalization, derealization, identity confusion and identity alteration with additional satisfaction of mood change and age regression. The patient met the diagnostic criteria for dissociative identity disorder in DSM-IV. She had distinct alternate personalities, each of which took complete control of her behavior, experienced episodes of severe amnesia during the domination of an alter, and was not influenced by either substance or any general medical condition.

The patient was a survivor of childhood emotional abuse and neglect from both parents. She remembered a time when she was left home alone at without food for several days. Treatment focused on stabilization, using affect management skills training and ego state therapy. The frequency of transitions to other altars gradually decreased, as did feelings of helplessness. The patient was discharged after the irritable alter remained absent for two continuous weeks.

\section{DISCUSSION}

Both patients' diagnosed were confirmed through a structured interview (SCD-D), and both patients demonstrated transitions to other personalities during one month of hospital- 
ization. Full psychological assessments, brain imaging studies, and laboratory tests were performed to rule out any influence of medical conditions, substances, or other psychiatric disorders. The two previously reported case reports of DID in Korea did not confirm the diagnosis through a structured interview, and in one case, alters were identified during hypnosis. ${ }^{6,7}$ Thus, our cases have better diagnostic validity than the previously reported cases in Korea. Additionally, our first patient demonstrated physiological changes during the alteration of identity, including horizontal eye movements, automatic hand writing with the opposite hand, and abnormal ECG findings.

Not surprisingly, both patients experienced repeated childhood physical abuse and neglect. Their violent alters appeared only when childhood traumatic event was remembered or recalled through emotional triggers, suggesting the role of the alter as a part of the ego created to deal with unacceptable experiences with overwhelming emotions on behalf of the original ego. ${ }^{8}$

During the last 15 years, no other of DID cases were diagnosed at our inpatient psychiatric unit prior to these two cases in 2014. Previously, the lower frequency of DID in Asia was believed to be due to a relatively higher prevalence of possession disorder, reflecting a strong cultural influence of polytheism and shamanism in the region. ${ }^{1}$ It is also likely that, in Korea, patients with possession disorder are more frequently seen in psychiatric practices, and as such, clinicians are less familiar with DID. ${ }^{9}$ Increased public awareness of mental illness and decreased psychological barriers to seeking psychiatric help ${ }^{10}$ might have contributed to recent increase in DID cases.

In sum, the recent social transition of Asian countries from traditionalism and collectivism toward Westernization and individualism, increasing awareness of dissociative phenomena in Korea, and possibly increasing recognition of child abuse and neglect recognition of child abuse and neglect ${ }^{11}$ might influence the increasing rates of DID. Mental health professionals from Asian regions who have rarely encountered patients with DID require specialized training to recognize and manage this chronic, often misdiagnosed, and difficult to treat condition.

\section{REFERENCES}

1. Varma VK, Bouri M, Wig NN. Multiple personality in India: comparison with hysterical possession state. Am J Psychother 1981;35:113-120.

2. International Society for the Study of Trauma and Dissociation. Guidelines for treating dissociative identity disorder in adults, third revision. J Trauma Dissociation 2011;12:115-187.

3. Ahsan MS, Mullick SI, Sobhan MA, Khanam M, Nahar JS, Salam MA, et al. Subtype of dissociative (conversion) disorder in two tertiary hospitals in Bangladesh. Mymensingh Med J 2010;19:66-71.

4. Chaturvedi SK, Desai G, Shaligram D. Dissociative disorders in a psychiatry institute in India-- a selected review and patterns over a decade. Int J Soc Psychiatry 2010;56:533-539.

5. Xiao Z, Yan H, Wang Z, Zou Z, Xu Y, Chen J, et al. Trauma and dissociation in China. Am J Psychiatry 2006;163:1388-1391.

6. Choe BM, Kim SH, Hahn HM, Yang CK, Eom YK. A case of dissociative identity disorder. J Korean Neuropsychiatr Assoc 1996;35:1487-1491.

7. Lee JS, Nam JH. A case of multiple personality disorder. Ment Health Res 1995;14:163-171.

8. van der Kolk BA, Fisler R. Dissociation and the fragmentary nature of traumatic memories: overview and exploratory study. J Trauma Stress 1995;8:505-525

9. Jung HJ. Clinical Characteristics of Inpatients Who Were Diagnosed as Possession Disorder [Dissertation]. Seoul: Hanyang University; 2013.

10. Korea Family Association for Mental Health Corp. Mental Illness Recognition Improvement Program: Media Monitoring. Seoul: Korea Family Association for Mental Health Corp; 2009.

11. Adityanjee, Raju GS, Khandelwal SK. Current status of multiple personality disorder in India. Am J Psychiatry 1989;146:1607-1610. 